\title{
Comparison of the effect of silicone gel sheets by thickness on excisional scars in pediatric and adolescent patients
}

\section{Min Ji Kim, Woo Beom Lee, Dong Ha Park}

Department of Plastic and Reconstructive Surgery, Ajou University Medical Center, Suwon, Korea
Background Selecting effective products among the various types of silicone gel sheets can be challenging for surgeons. Therefore, we assessed the effect of silicone gel sheet thickness on surgical scars in pediatric and adolescent patients.

Methods From December 2017 to May 2018, we identified patients aged 1-19 years who underwent excision. Among these patients, those who were prescribed 0.3-mm or 1.0-mm-thick silicone sheets were selected. Scars were subjectively evaluated using a questionnaire consisting of seven items. Objective evaluation was performed by two plastic surgeons using the Vancouver Scar Scale (VSS).

Results The mean age of the 49 selected patients was 9.78 years. The patients were divided into two groups according to the thickness of the silicone gel sheet used $(0.3 \mathrm{~mm}$ vs. $1.0 \mathrm{~mm}$ ). Objective evaluation of the patients' scars revealed more favorable results in the $0.3 \mathrm{~mm}$ group than in the $1.0 \mathrm{~mm}$ group $(\mathrm{P}=0.010)$. Multivariate analysis of VSS scores indicated that the resulting scars in cases involving the trunk were of poorer quality than those involving facial areas $(\mathrm{P}=0.015)$. Additionally, favorable (i.e., belowaverage) VSS scores were significantly less likely in patients with longer scars (odds ratio, 0.896; 95\% confidence interval, $0.834-0.963 ; \mathrm{P}=0.003$ ) or thicker silicone sheets (odds ratio, 0.085; 95\% confidence interval, 0.011-0.699; $\mathrm{P}=0.019$ ).

Conclusions The use of thinner silicone gel sheets in children and adolescents resulted in better scars according to subjective evaluations, underscoring the importance of compliance in pediatric patients. The type of operation and surgical lesion should also be considered when planning the management of surgical scars.

Keywords Cicatrix / Silicone gel / Hypertrophic / Pediatrics

\section{INTRODUCTION}

In addition to aesthetic concerns, surgical scars may be associated with several physical symptoms, including hypertrichosis, dyshi-

Received: Nov 7, 2021 Revised: Nov 26, 2021 Accepted: Nov 30, 2021 Correspondence: Dong Ha Park Department of Plastic and Reconstructive Surgery, Ajou University Medical Center, 164 World cup-ro, Yeongtong-gu, Suwon 16499, Korea

Tel: +82-31-219-5614, Fax: +82-31-219-5610, E-mail: growhand@hanmail.net

Copyright @ 2022 The Korean Society for Aesthetic Plastic Surgery.

This is an Open Access article distributed under the terms of the Creative Commons Attribution Non-Commercial License (https://creativecommons.org/licenses/by-nc/4.0/) which permits unrestricted non-commercial use, distribution, and reproduction in any medium, provided the original work is properly cited. $\quad w w w . e-a a p s . o r g$ drosis, pain, pruritus, dysesthesia, and functional impairments such as contracture [1,2]. Research on scar quality and management has been conducted using various methods. However, due to the lack of a reliable scar assessment tool, uneven patient compliance, and difficulty in obtaining consent from caregivers in pediatric and adolescent patients, the study of these groups has been limited, except for a few studies on scars following cleft lip surgery or burn injury [3].

Various surgical techniques are used to reduce the widening of scars. These range from commonly used methods, such as Z-plasty or local flaps, to new techniques, such as the dermal splinting technique, in which the dermal structure of the scar is subjected to tension [4]. Laser treatments are also very prevalent after surgical excision. One recent study revealed that, even in the early stages of 
scar formation, laser therapy improved erythema, hyperpigmentation, and hypertrophy [5]. Steroid injections are frequently used for hypertrophic scars or keloid scars, and a recent study found that a cocktail therapy of bleomycin and verapamil was also effective for severe keloid scar management [6]. Various adjunctive methods for scar management have also been tried, such as cream gels containing silicone, gel sheets, and ointments containing silicone gel [7]. Among these various techniques, silicone gel sheets are frequently prescribed by plastic surgeons after surgical procedures and for patients with posttraumatic scars. Moreover, in pediatric patients, non-invasive methods such as silicone gel sheets or ointment are preferred over invasive procedures such as laser or steroid injections, due to pain and control of patient movement during the procedure.

Since the early 1980s, following the first study by Perkins et al. [8], a wide range of silicone-based products (silicone gel sheeting, tapes, bandages, cream containing silicone oil, and self-drying silicone gel) have been widely used in the treatment of hypertrophic scars and keloids. In several previously published studies, the application of silicone gels provided improvements in redness, itching, texture, and thickness of hypertrophic scars and keloids in 60\% to $100 \%$ of patients. Silicone gel sheets were reported by O'Brien and Jones [9] in 2013 to significantly reduce the incidence of hypertrophic scarring among high-risk individuals, increase scar elasticity, and reduce redness [9].

Based on the effectiveness of silicone gel sheets in treating various scars, there have been many market trials of diverse types of scar sheets. Different companies distinguish their products with distinctive compositions, shapes, sizes, textures, elasticity, or adhesiveness of the sheets. As a result of this overwhelming amount of information regarding silicone scar sheets, it is often challenging for plastic surgeons to choose the most appropriate product. Various factors need to be considered, such as the anatomical site, scar length, and patient compliance. There is a need for clear recommendations to guide the choice of scar product, particularly for pediatric patients. Therefore, reliable information regarding the different effects of available products based on their characteristics would be useful to physicians. Among the various properties of silicone scar sheets, we focused on the thickness of the sheet. In our study, we assessed the effects of silicone gel sheets on surgical scars, depending on the thickness of the sheet, in pediatric and adolescent patients. Using objective and subjective scar assessment tools, we demonstrated the effect of silicone gel sheets on scar quality.

\section{METHODS}

We retrospectively selected and followed patients who underwent silicone gel sheet application after excision of benign tumors or benign skin masses between December 2018 and May 2019 at Ajou University Hospital, Suwon, Korea. Patients were between 1 and 19 years of age and their baseline information was collected through chart reviews.

The silicone sheets used were ScarSense-alpha (1.0 mm; CGbio, Seoul, Korea) and BAP Scar Care (0.3 mm; BAP Medical BV, Apeldoorn, Netherlands). In the outpatient clinic, silicone gel sheets were randomly prescribed to patients and the patient or caregiver was instructed to apply the sheet for more than 8 hours a day (continuously or in divided time periods); the usage time and method of application for the silicone gel sheets were the same for each group.

Scars were assessed approximately 3 months postoperatively. Objective evaluation using the Vancouver Scar Scale (VSS) was conducted by two plastic surgeons (MJK and WBL). The subjective assessment for scar quality was obtained from patients who filled out a pre-made questionnaire. For patients $<7$ years old, the questionnaire was completed by their parents or guardians. Since this study involved children and adolescents, permission was obtained from the patients' parents or guardians at the time of visitation and the questionnaire was completed to score the subjective evaluation of the scar. For the VSS, two physicians scored the clinical photographs. The VSS scoring system included evaluations of pigmentation, vascularity, pliability, height, and length, for a total score of 15 points. When the length of the scar was $<3 \mathrm{~cm}$, we evaluated it based on the middle portion of the scar. However, when the length of the scar was $>3 \mathrm{~cm}$, we evaluated it by dividing the scar equally into three parts and then calculated the average score. In the subjective evaluation, a seven-item questionnaire was completed by patients or their caregivers and the contents of this questionnaire were used to evaluate pain, itching, scar color, stiffness, thickness, irregularity, and overall satisfaction in the weeks preceding the visit. The satisfaction level of each question comprised 10 points with a total score of 60 points. The patient group included infants as well as adolescents, so parents were asked to complete the questionnaire if the patient was unable to fill it out (Figs. 1, 2).

This study was performed in accordance with the principles of the Declaration of Helsinki. The study and all its protocols were approved by the Institutional Review Board of Ajou Medical Center (approval no. MED-MDB-20-109). The need for informed consent was waived by the institutional review board because of the retrospective nature of the study. However, photographic authorization and release consent were obtained from all participants and their parents.

Continuous variables were presented as means and standard deviations and were compared using the t-test. Categorical variables were presented as frequencies and percentages and were compared using the chi-square and Fisher exact tests. To analyze the factors associated with favorable scarring, we defined a favorable scar as a VSS score below the average. The factors for favorable scarring were first identified through univariate analysis, and significant variables 

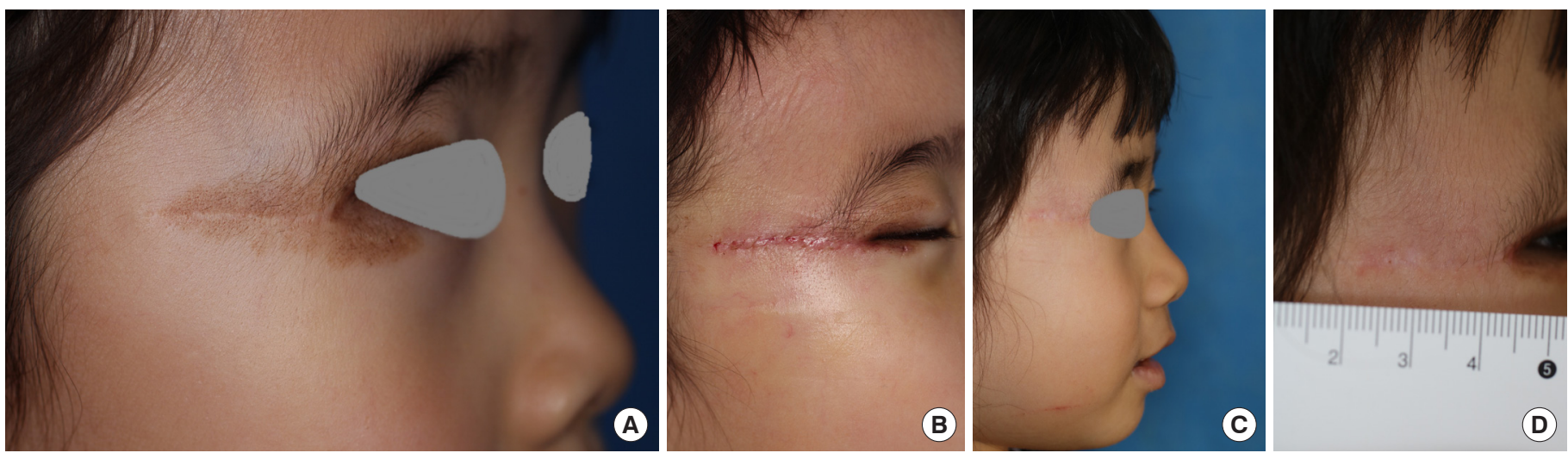

Fig. 1. Representative case of a patient treated with a $0.3-\mathrm{mm}$ silicone gel sheet on the facial area after excision of a nevus. A 4-year-old female patient underwent excision of a nevus in the right lateral orbital area. A compound nevus was confirmed by histological examination, and after suture removal, a $0.3-\mathrm{mm}$ silicone gel sheet was used for 3 months. The patient's progress was monitored in the outpatient clinic. The Patient Assessment Score completed by her parents was 6 points, and the Vancouver Scar Scale score evaluated at the outpatient clinic was 2 points. (A) Preoperative views. (B) Image immediately after suture removal. (C, D) Images after 3 months of using a 0.3-mm silicone sheet.
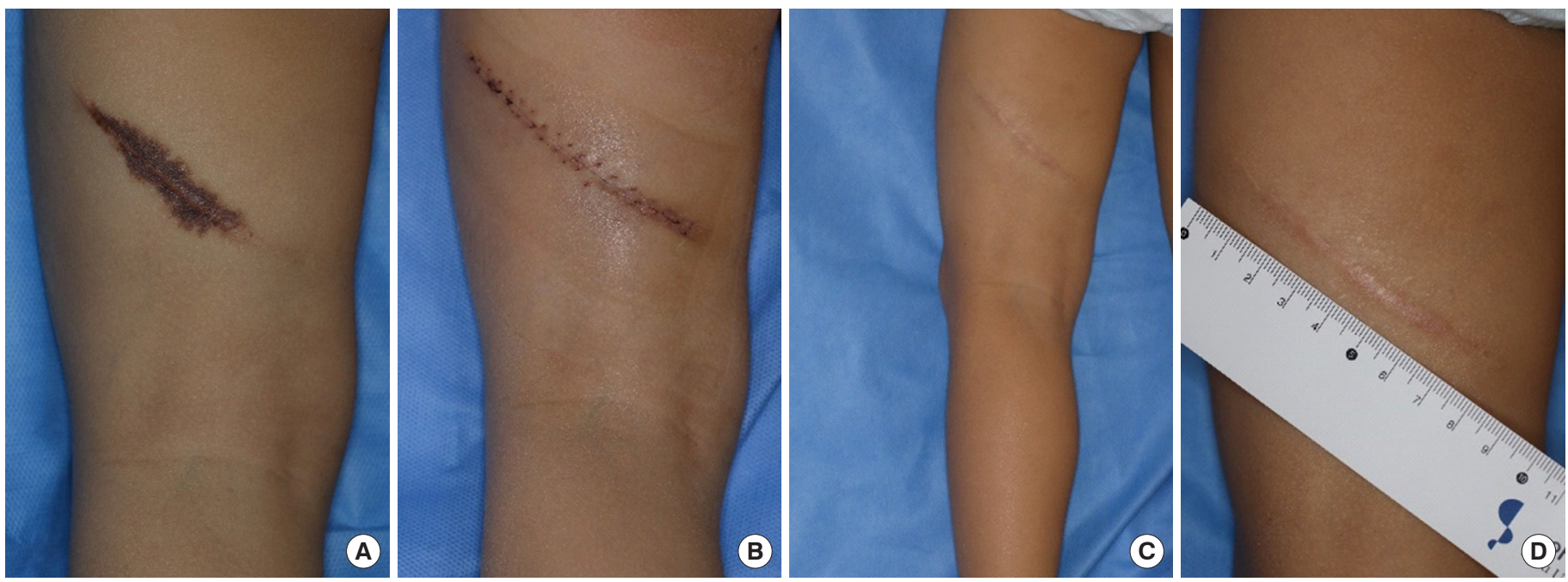

Fig. 2. Representative case of a patient treated with a 1.0- $\mathrm{mm}$ silicone gel sheet on the extremity after excision of nevus. A 6-year-old female patient underwent excision of a nevus on the posterior surface of the left thigh. A compound nevus was also confirmed through histological examination. After suture removal, a $1.0 \mathrm{~mm}$ silicone gel sheet was used for 3 months, and the patient's progress was monitored in the outpatient clinic. The Patient Assessment Score completed by her parents was 10 points, and the Vancouver Scar Scale score evaluated at the outpatient clinic was 5 points. (A) Preoperative views. (B) Image immediately after suture removal. (C, D) Images after 3 months of using a 1.0 mm silicone sheet.

$(\mathrm{P}<0.05)$ were included in the multivariate analysis. Backward-stepwise logistic regression analysis was performed to identify related factors for favorable scarring. All statistical analyses were performed using SPSS version 19.0 (IBM Corp., Armonk, NY, USA). A P-value of $<0.05$ was considered to indicate statistical significance.

\section{RESULTS}

A total of 49 pediatric and adolescent patients, with a mean age of 9.78 years (range, 11 months- 17 years), fulfilled the inclusion cri- teria and comprised the analytical cohort of the study. The majority of patients in this study were female (female: $71.4 \%$, male: $28.6 \%$ ). The average follow-up period was 331.1 days ( 330 days for the 0.3$\mathrm{mm}$ group and 332.6 days for the $1.0-\mathrm{mm}$ group). The most prevalent type of pathology for the excised mass was compound nevus (57.1\%), followed by nevus sebaceous (22.5\%). The facial area was the most common site of operation in this cohort (59.2\%), followed by the upper extremity (18.4\%) and trunk (12.2\%). The mean length of the scars after surgery was $45.10 \pm 38.91 \mathrm{~mm}$. The mean objective (VSS: total 15 points) and subjective (Patient Assessment Score 
[PAS]: total 60 points) scar assessment scores were $3.73 \pm 1.98$ points and $8.86 \pm 6.08$ points, respectively (Table 1 ).

Patients were divided into two groups according to the thickness of the silicone gel sheet used: $0.3 \mathrm{~mm}$ or $1.0 \mathrm{~mm}$. Local flap operations were used significantly more often in the $0.3-\mathrm{mm}$ sheet group ( $25 \%$ in the $0.3-\mathrm{mm}$ sheet vs. $4 \%$ in the $1.0-\mathrm{mm}$ sheet, $\mathrm{P}=0.036$ ). In other operation types, there was no significant difference between the two groups. In addition, the thinner silicone gel sheet was used more frequently when the facial area was involved (75\% in the $0.3-\mathrm{mm}$ sheet vs. $44 \%$ in the $1.0-\mathrm{mm}$ sheet, $\mathrm{P}=0.027$ ). The scar length after excisional surgery was observed to be longer in the 1.0-mm silicone sheet group, but the difference was not statistically significant $(42.67 \pm 28.34 \mathrm{~mm}$ in the $0.3-\mathrm{mm}$ sheet group vs. $65.08 \pm 44.75 \mathrm{~mm}$ in the $1.0-\mathrm{mm}$ sheet group, $\mathrm{P}=0.085$ ) (Table 2).

The results of objective and subjective scar assessments are shown in Table 3. In the objective assessment using the VSS, low scores indicated favorable scars. Our analysis revealed that the thinner silicone gel sheet had significantly more favorable outcomes (3.25 \pm 1.48 in the $0.3-\mathrm{mm}$ group vs. $4.20 \pm 2.30$ in the 1.0 - $\mathrm{mm}$ group, $\mathrm{P}=0.010$ ). In addition, subjective analysis of the scars using the PAS scores, revealed that the condition of the scars was more fa-

Table 1. Patient demographics and operative details

\begin{tabular}{lc}
\hline Variable & Value $(\mathrm{n}=49)$ \\
\hline Age lyr) & $9.78 \pm 9.40$ \\
Sex & \\
Female & $35(71.43)$ \\
Male & $14(28.57)$ \\
Pathology & \\
Epidermal cyst & $1(2.04)$ \\
Pilomatricoma & $1(2.04)$ \\
Lipoma & $1(2.04)$ \\
Compound nevus & $28(57.14)$ \\
Intradermal nevus & $3(6.12)$ \\
Nevus sebaceous & $11(22.45)$ \\
Junctional nevus & $2(4.08)$ \\
Others & $2(4.08)$ \\
Location of scar & \\
Face & $29(59.18)$ \\
Lower extremity & $3(6.12)$ \\
Upper extremity & $9(18.37)$ \\
Back & $2(4.08)$ \\
Trunk & $6(12.24)$ \\
Length of scar (mm) & $45.10 \pm 38.91$ \\
Vancouver Scar Scale (total 15 points) & $3.73 \pm 1.98$ \\
Patient Assessment Scale (total 60 points) & $8.86 \pm 6.08$ \\
\hline
\end{tabular}

Values are presented as mean \pm SD or number $(\%)$.

a) Hemangioma, skin tag. vorable in the $0.3-\mathrm{mm}$ gel sheet group than in the $1.0-\mathrm{mm}$ gel sheet group, but the difference was not statistically significant $(8.00 \pm 5.77$ in the 0.3 -mm group vs. $9.68 \pm 6.36$ in the 1.0 -mm group, $\mathrm{P}=0.634$ ). Even when the perfect score of the two evaluation methods was converted to 100 points and corrected, the result was equivalent to those before conversion. To minimize the gap between VSS and PAS, we also evaluated the absolute value of the difference between VSS and PAS and no statistical significance was observed (Table 3).

To identify the factors that affected favorable scarring, we performed multivariate analysis. The results are shown in Table 4. Multivariate analysis was performed using factors identified as significant in univariate analyses; thus, age, sex, type of lesion, type of operation, type of gel sheet, and length of scar were used for the analysis. For this study, the definition of a favorable scar was a belowaverage VSS score (3.73 points from a total of 15 points), and a positive odds ratio $(\mathrm{OR}>1)$ meant that the variable had favorable effects on the scar. Age and sex did not have a statistically signifi-

Table 2. Comparative analysis of scar characteristics according to the thickness of the silicone gel sheet

\begin{tabular}{lccc}
\hline Variable & $0.3-\mathrm{mm}$ gel sheet & 1.0- $\mathrm{mm}$ gel sheet & P-value \\
\hline No. of patients & 24 & 25 & \\
Age lyr) & $8.88 \pm 5.68$ & $8.48 \pm 7.06$ & 0.210 \\
Type of operation & & & \\
Simple excision & $18(75.0)$ & $22(88.0)$ & 0.240 \\
Tissue expander & 0 & $2(8.0)$ & 0.157 \\
Local flap & $6(25.0)$ & $1(4.0)$ & $0.036^{\text {a) }}$ \\
Site of operation & & & \\
Face & $18(75.0)$ & $11(44.0)$ & $0.027^{\mathrm{a})}$ \\
Lower extremity & $2(8.3)$ & $1(4.0)$ & 0.413 \\
Upper extremity & $2(8.3)$ & $7(28.0)$ & 0.076 \\
Back & 0 & $2(8.0)$ & 0.527 \\
Trunk & $2(8.3)$ & $4(16.0)$ & 0.157 \\
Length of scar $(\mathrm{mm})$ & $42.67 \pm 28.34$ & $65.08 \pm 44.75$ & 0.085 \\
\hline
\end{tabular}

Values are presented as mean \pm SD or number $(\%)$.

${ }^{\text {a) }}$ Statistically significant.

Table 3. Comparison of scar assessment scores of patients treated with different silicone gel sheets

\begin{tabular}{lccc}
\hline Score & 0.3-mm gel sheet & 1.0-mm gel sheet & P-value \\
\hline VSS score (total 15 points) & $3.25 \pm 1.48$ & $4.20 \pm 2.30$ & $0.010^{\text {bl }}$ \\
PAS score (total 60 points) & $8.00 \pm 5.77$ & $9.68 \pm 6.36$ & 0.634 \\
Calculated VSS & $21.67 \pm 9.88$ & $28.00 \pm 15.40$ & $0.010^{\text {bl }}$ \\
Calculated PAS $^{\text {a) }}$ & $13.33 \pm 9.63$ & $16.13 \pm 10.061$ & 0.634 \\
$\Delta$ Calculated PAS-VSS & $8.82 \pm 6.15$ & $11.87 \pm 7.21$ & 0.333
\end{tabular}

Values are presented as mean \pm SD.

VSS, Vancouver Scar Scale; PAS, Patient Assessment Score.

${ }^{\text {al }}$ Converted to 100 points; ${ }^{b}$ Statistically significant. 
Table 4. Multivariate logistic regression analysis of factors associated with a favorable scar (a below-average VSS score) after benign mass excision

\begin{tabular}{lcl}
\hline Variable & Odds ratio (95\% Cl) & P-value \\
\hline Age & $0.865(0.743-1.007)$ & 0.061 \\
Sex & $1.601(0.207-12.349)$ & 0.652 \\
Site of lesion & 1.000 & \\
Face & $0.002(0.000-0.286)$ & $0.015^{\text {a) }}$ \\
Trunk & $0.015(0.000-2.152)$ & 0.097 \\
Upper extremity & $1.349(0.026-70.144)$ & 0.882 \\
Lower extremity & $0.111(0.000-50.609)$ & 0.482 \\
Back & $2.832(0.175-45.828)$ & 0.464 \\
Type of operation surgical excision & $0.896(0.834-0.963)$ & $0.003^{\text {a) }}$ \\
Length of scar & $0.085(0.011-0.699)$ & $0.019^{\text {a) }}$ \\
\hline Type of silicone gel sheet & & \\
\hline
\end{tabular}

VSS, Vancouver Scar Scale; Cl, confidence interval.

al Statistically significant.

cant effect on the VSS score (age: OR, $0.865 ; 95 \%$ confidence interval [CI], 0.743-1.007; $\mathrm{P}=0.061$ and sex: OR, 1.601; 95\% CI, 0.20712.349; $\mathrm{P}=0.652$ ). However, significantly higher VSS scores (i.e., poorer-quality scars) were found in cases involving the trunk than in cases involving the facial area (OR, 0.002; 95\% CI, 0.000-0.286; $\mathrm{P}=0.015$ ). Although not statistically significant, there was a similar trend in the group that underwent surgery on the upper extremity and back, but not for the lower extremity. In addition, no statistically significant difference was found between cases that received simple excision and cases involving other surgical procedures (e.g., a local flap or tissue expander) (OR, 2.832; 95\% CI, 0.175-45.828; $\mathrm{P}=0.464)$. However, unfavorable associations were found for longer scars (OR, 0.896; 95\% CI, 0.834-0.963; $\mathrm{P}=0.003)$ and thicker silicone gel sheets (OR, 0.085; 95\% CI, 0.011-0.699; $\mathrm{P}=0.003$ ) (Table 4).

\section{DISCUSSION}

Several scar management products, including silicone gels, are used in various clinical situations; of these products, silicone gel sheets are the most widely used. The effectiveness of silicone gel sheets has been demonstrated in many previous studies.

Perkins et al. [8] first observed the potential usefulness of silicone gel sheets for the treatment of burn scars and contractures in the early 1980s.

Subsequently, several randomized controlled trials (Carney et al.) provided strong evidence that silicone gel sheets are effective in the treatment of hypertrophic scars [10].

In 2010, a prospective study by Bianchi et al. [11] used the Patient and Observer Scar Assessment Scale (POSAS) to evaluate the outcomes of the healing process of posttraumatic and surgical fa- cial scars that were treated with silicone gel; they reported satisfactory management of posttraumatic and surgical facial scars with self-drying silicone gel evaluated using the POSAS.

Some studies have also demonstrated the effectiveness of silicone gel sheets in specific races. In 2010, Rhee et al. [12] analyzed 40 patients who were either treated with or without silicone gel sheets and demonstrated the effectiveness of silicone gel sheets in Asian patients.

The mechanism of action of silicone-based products in scar management has not been fully elucidated, but there is consensus on its general action. The silicone gel sheet occludes and holds the periphery of hypertrophic scars and reduces scar formation by reducing tension around the scar [13]. Therefore, hydration and occlusion are the primary mechanisms underlying the therapeutic effect of silicone on scars [14]. Decreased evaporation of water from the skin with increased hydration of the stratum corneum and occlusion results in a favorable scar. There are also reports that silicone sheets can affect the activity and growth factor production of cultured fibroblasts in hypertrophic scars and keloids [15].

Generally, as the thickness of the gel sheet increases, the tension applied around the scar can be effectively maintained; therefore, thicker sheets are often expected to provide better scar management. While many studies have discussed the effects of silicone gel sheets themselves, few studies have compared the effects of sheet thickness.

Patient cooperation is also very important in scar management because patients may experience discomfort for an extended time. Patients' discomfort may be exacerbated by thicker silicone gel sheets. Therefore, it is important to determine whether there is a meaningful difference in the effect according to the thickness of the silicone gel sheet used. This study was performed to address this issue.

It was also meaningful that this study limited the enrolled patient group to Asian children and adolescents, who are relatively less compliant with scar management, so that the effect of silicone gel sheets can be observed in these age groups.

The results of this study revealed that the PAS and the VSS scores assigned by medical staff were lower in the $0.3-\mathrm{mm}$ thickness group than in the $1.0-\mathrm{mm}$ thickness group. In other words, the thinnersheet group obtained better scar management results.

To investigate the reasons for these results, we first considered the difference in compliance according to the thickness of the silicone gel sheet. Management of scars can extend over a long period, often for several months; therefore, patient compliance with treatment is very important. Generally, a thicker silicone sheet material increases the discomfort experienced by the patient, including pressure and limitation of movement. When a thick silicone sheet is used, patient compliance declines. Therefore, in our study, the thicker silicone gel sheets may have achieved worse results due to reduced patient compliance.

A second factor in the observed differences was the length of the 
surgical site. Longer surgical sites are generally more difficult to manage than smaller ones, and this principle also applies to scar management. Additionally, scar management requires long-term care and treatment.

Third, the surgical site can affect the differences observed in scar outcomes. Long and short scars may coexist in the same facial area, but the 1.0-mm thickness group had longer scars and were more likely to have undergone operations on the extremities and trunk, which were subjected to more tension than the face. In outpatient settings, doctors generally prescribe thicker silicone gel sheets for relatively long surgical sites or areas of high tension, such as the abdomen and arms. The group with long scars included patients who underwent considerable surgery in tension-prone areas. It is well known that tension increases the likelihood of postoperative scarring, which may explain the differences in outcomes based on site.

There were some limitations to our study. First, patient compliance is an important factor that affects the outcome of scars, especially in pediatric and adolescent patients. In our institution, we educated patients and their caregivers using the same guidelines, aiming to minimize the compliance gap. However, depending on the patients, the daily gel sheet usage time and the time taken before changing to a new gel sheet could not be completely controlled, and these factors were not analyzed in the study. Therefore, compliance-related factors should be included in future studies.

Second, different surgical sites were used. The surgical site is an important factor to consider in scar management because relevant variables change with each body part. Factors such as tension due to movement, external stimulation, and the ease of applying the gel sheet vary even if a similar surgical method is used or the length of the scar is similar. When reviewing the results of multivariate logistic regression analysis, the facial area was found to have a statistically significant effect on favorable scars compared to the trunk region, whereas the lower extremity had more favorable scars than the facial area, even though this was not statistically significant. In this study, consistent results may not have been obtained due to the small number of patients for each body part. Therefore, future studies with a larger number of patients are required to clarify this relationship.

Third, patients in different age groups were included. Unlike previously published studies, our study is meaningful in that it was limited to children and adolescents. However, the proportion of children $<7$ years old, who may be relatively less compliant, was $55 \%$ (27 patients), which was much higher than those of other age groups and could have influenced the results.

Fourth, there were differences in some characteristics of the products, as they were produced by different manufacturers. In this study, we focused on the effect of the thickness of the silicone gel sheets; therefore, we selected two silicone gel sheets from different companies. However, in addition to the thickness of the sheet, various factors, such as the pattern of the silicone contact surface, the degree of adhesion, and viscosity can affect the results of scars. Therefore, in future studies, it would be preferable to compare products produced by the same manufacture with similar characteristics other than thickness.

Finally, selection bias could have occurred since there were products preferred by the attending surgeon, depending on the surgical site, and this may have also affected the results. In this study, an association between thickness and favorable scars was identified. However, it will be necessary to verify the results of this study through a double-blind, randomized, controlled study in the future.

Future prospective studies that are limited to a specific age group and surgical site and include the evaluation of scar conditions several times during a fixed follow-up period, would provide meaningful results.

In conclusion, we demonstrated that the use of thinner silicone gel sheets in children and adolescent groups resulted in better scars according to subjective evaluations, in part because compliance is particularly important in pediatric patients. This result was also confirmed by multivariate logistic regression analysis. Additionally, the length of the surgical wound can affect the quality of the scar, and the type of operation and surgical lesion should be considered together in the management of surgical scars. Our results show that proper selection of thin silicone gel sheets leads to better scars in pediatric and adolescent patients.

\section{NOTES}

\section{Conflict of interest}

Dong Ha Park is an editorial board member of the journal but was not involved in the peer reviewer selection, evaluation, or decision process of this article. No other potential conflicts of interest relevant to this article were reported.

\section{Ethical approval}

The study was approved by the Institutional Review Board of Ajou Medical Center (IRB No. MED-MDB-20-109) and performed in accordance with the principles of the Declaration of Helsinki.

\section{Patient consent}

The all participants and their parents provided written informed consent for the publication and the use of patients' images.

\section{ORCID}

Min Ji Kim

Woo Beom Lee

Dong Ha Park

https://orcid.org/0000-0002-0723-4382

https://orcid.org/0000-0002-5333-5424

https://orcid.org/0000-0002-8024-5846

\section{REFERENCES}

1. Rabello FB, Souza CD, Farina Junior JA. Update on hypertrophic scar 
treatment. Clinics (Sao Paulo) 2014;69:565-73.

2. Tebble NJ, Adams R, Thomas DW, et al. Anxiety and self-consciousness in patients with facial lacerations one week and six months later. Br J Oral Maxillofac Surg 2006;44:520-5.

3. Mundy LR, Miller HC, Klassen AF, et al. Patient-reported outcome instruments for surgical and traumatic scars: a systematic review of their development, content, and psychometric validation. Aesthetic Plast Surg 2016;40:792-800.

4. Namgoong S, Jung JA, Kim DY, et al. Revision of a widened scar using dermal splinting technique. Arch Aesthetic Plast Surg 2015;21:75-80.

5. Jang JY, Han JH, Yoon KC, et al. Early management of scars using a 532-nm Nd:YAG laser. Arch Aesthetic Plast Surg 2017;23:62-7.

6. Park JH, Chang H. Cocktail therapy including bleomycin and verapamil as a promising treatment choice for keloid scars. Arch Aesthetic Plast Surg 2020;26:99-104.

7. Kim JS, Hong JP, Choi JW, et al. The efficacy of a silicone sheet in postoperative scar management. Adv Skin Wound Care 2016;29:414-20.

8. Perkins K, Davey RB, Wallis KA. Silicone gel: a new treatment for burn scars and contractures. Burns Incl Therm Inj 1983;9:201-4.

9. O’Brien L, Jones DJ. Silicone gel sheeting for preventing and treating hypertrophic and keloid scars. Cochrane Database Syst Rev 2013;2013: CD003826.

10. Carney SA, Cason CG, Gowar JP, et al. Cica-Care gel sheeting in the management of hypertrophic scarring. Burns 1994;20:163-7.

11. Bianchi FA, Roccia F, Fiorini P, et al. Use of Patient and Observer Scar Assessment Scale for evaluation of facial scars treated with self-drying silicone gel. J Craniofac Surg 2010;21:719-23.

12. Rhee SH, Koh SH, Lee DW, et al. Aesthetic effect of silicone gel on surgical scars in Asians. J Craniofac Surg 2010;21:706-10.

13. Mustoe TA. Evolution of silicone therapy and mechanism of action in scar management. Aesthetic Plast Surg 2008;32:82-92.

14. Chang CC, Kuo YF, Chiu HC, et al. Hydration, not silicone, modulates the effects of keratinocytes on fibroblasts. J Surg Res 1995;59:705-11.

15. Borgognoni L. Biological effects of silicone gel sheeting. Wound Repair Regen 2002;10:118-21. 